\title{
Insulin technique
}

\section{Sanjay Kalra', Yashdeep Gupta ${ }^{2}$}

Sri Lanka Journal of Diabetes, Endocrinology and Metabolism 2015; 5: 85-90

\begin{abstract}
Insulin is an important treatment modality of treating diabetes and insulin technique; is a vital component of the insulin prescription and of diabetes care. A thorough knowledge regarding insulin preparations, devices, injecting techniques and the problems encountered with insulin use are essential for both health care providers and the patients using insulin. This review highlights salient features of insulin technique, choice of delivery devices and storage of insulin in resource challenged settings.
\end{abstract}

Key words: insulin technique, injection site, lipohypertrophy, disposal, bio-psychosocial.

\section{Introduction}

An appropriate insulin prescription, including the right choice of insulin preparation, the regime and the dosage, is important for the success of diabetes management; so is the correct insulin injection technique. Inappropriate injection technique has the potential not only to limit the utility of insulin, but also to harm the patient. Similar to the strategy of choosing an insulin prescription for a particular patient, insulin technique should also be decided and explained according to the person's requirements.

Many factors influence the choice of injection technique in persons with diabetes (Table 1). Injection technique is not limited to the injection itself. It comprises the entire process of pre-injection assessment, counseling and motivation, selection of device and explanation of its use, selection and preparation of the injection site, $t$ preparation of the insulin/insulin device, and the actual method of injecting. Injection technique does not end with delivery of insulin into the subcutaneous space and needs attention for trouble shooting, pain minimization, management of needle phobia and local injection site reactions. It also includes tips on insulin storage, travelling with insulin, needles/syringe hygiene and disposal of injection-related accessories. Insulin technique education is not a one-time intervention and it is an on-going process of insulin therapy in diabetes care. The aim of this article is to update the knowledge of health care providers regarding these important pre-requisites for proper injection technique.

\section{Pre injection assessment}

A thorough clinical (biopsychosocial) and environ-
Table 1. Factors influencing insulin injection technique

\section{Biological factors}

- Choice of site of injection

- Depth of subcutaneous tissue

- Dexterity

- Visual health

- Auditory health

\section{Psychological factors}

- Fear of insulin

- Fear of needles

- Patient motivation

\section{Social factors}

- Clinician's knowledge

- Clinician's time

- Patient's learning skills

mental assessment must be done while suggesting insulin therapy. Biomedical factors such as visual, auditory or upper limb impairment, which may limit ability to self injection, must be considered (1). Psychological barriers to insulin must be explored and addressed and sociocultural issues respected $(2,3)$. Availability of facilities for insulin storage and disposal must be assessed and modified if required. Psychological insulin resistance, driven by personal anxiety and fuelled by misinformed

${ }^{1}$ Department of endocrinology, Bharti hospital, Karnal, India, ${ }^{2}$ Department of medicine, government medical college, Chandigarh, India. 
advice from friends and relatives, is common in South Asia. Such self-erected barriers to insulin therapy must be bridged before insulin technique can be taught successfully $(2,3)$. This is best done through a process of shared decision making, which promotes patient empowerment. Children, adolescents, antenatal women and the elderly need different counseling strategies from adults and must be approached in a manner, which makes them comfortable (4).

\section{Insulin storage}

Specific storage guidelines are usually mentioned by manufacturers in pack inserts. Insulin should be stored at $2-8^{\circ} \mathrm{C}$ in refrigerators, but can be kept at room temperature $\left(15-25^{\circ} \mathrm{C}\right)$ for up to a month (4). Various methods of insulin storage have been suggested for resource-constrained settings (Table 2).

Table 2. Alternative methods of insulin storage

\section{In hot climes}

- In bowl half filled with water

- In earthen ware pitcher, half filled with water

- In a shallow hole, dug in the ground

- In thermo cool boxes with dry ice

\section{In very cold climes}

- Wrapped in multiple layers of woolen cloth

- In thermo cool boxes

- In insulin wallets

\section{Travel with insulin}

While travelling by surface transport, insulin can be kept in flask with ice or in a hand bag. It should never be kept in the glove compartment of a car or in a locked car during summer. During air travel, insulin should be kept in hand baggage (4).

\section{Device selection}

Choosing the correct device, appropriate to the person's needs, is vital for successful insulin therapy. The biopsychosocial model of health, which gives equal importance to biological (biomedical), psychological and social aspects of health, can be used to select an appropriate insulin delivery device. Some of the biopsychosocial factors, which inform choice of delivery device, are listed in Table 3.
Table 3. Alternative methods of insulin storage

\section{Biological factors}

- Manual dexterity

- Visual acuity

- Auditory acuity

- Magnitude of insulin dose required

- Adjustment of insulin dose required

\section{Psychological factors}

- Personal preference of pen vs. vial/ syringe

\section{Social factors}

- Financial burden

- Need for privacy while injecting

- Need for injection while at work/in school/ during travel

- Lifestyle

\section{Product-related factors}

- Number of units that a pen/syringe can hold

- Largest dose that can be injected

- Minimum dose that can be changed (increments of half, one, or two units)

- Indicators for adequacy of insulin left in pen

- Scope for corrective measures if wrong dose is dialed

\section{Pre-injection checklist}

Prior to injecting insulin, one must check the delivery device, the insulin and compatibility of all related accessories.

Insulin vials (U40 or U100) must be compatible with their syringes, which can be recognized by their colors and scales. U100 insulin syringes have an orange cover and black scale markings denoting two units each, while U40 syringes have a red cover and red scale markings of one unit each. In general, thinner 31 gauge needles are preferred. Usually the needle length is $6 \mathrm{~mm}$ or $8 \mathrm{~mm}$ and there is no advantage of using longer needles (5).

Insulin pens can be reusable or disposable. One must ensure that cartridges, reusable pens and needles are 
compatible with each other. Pen needles may vary from $4 \mathrm{~mm}$ to $8 \mathrm{~mm}$ in length and 30 gauges to 32 gauges in thickness (5).

\section{Needle length in specific patient populations}

Modern 4 to $6 \mathrm{~mm}$ long needles can be used for nearly everyone. Skin fold should be raised only if longer needles are being used or if the injection is being administered to a very slim person (BMI $<20 \mathrm{~kg} / \mathrm{m}^{2}$ ) or toddlers $(4,5,6)$.

However, making a skin fold (raised using the thumb and index finger), apart from avoiding intramuscular injection, is helpful in creating diffuse depot of insulin that facilitate uniform absorption. The skin should not be squeezed so tightly that could cause pain or blanching and should be released only after the drug has been injected and the needle withdrawn (4). Injecting at $45^{\circ}$ angle is another option in these cases.

\section{Injection sites}

Insulin injections should be administered into the subcutaneous tissue to ensure the most reliable and consistent absorption of insulin. Intramuscular injections speeds up absorption and can lead to unexpected hypoglycemia. On the other hand, intradermal injections are more painful, can lead to leakage of insulin from the puncture site, can lead to sterile abscesses and also enhance immune reactions to insulin due to lymphocyte stimulation and should be avoided. The absorption rates are nearly same from the superficial fat layer as is from deep layers near the muscle fascia (6).

Routinely, the abdomen and anterior thigh are used to inject insulin. The lower abdomen, extending from a line drawn 1 inch above the umbilicus to a line connecting the anterior superior iliac spines, may be used. However, the medial part of this space, extending 2 inches from the umbilicus on both sides, should be avoided. The absorption rates are fastest from abdomen. However, in case of multiple dose injection regimens, thigh is the preferred alternative site for injections. The preferred injection sites over the thigh are in the outer and anterior part of the mid one third of the area between anterior superior iliac spine and the patella. The medial part of the thigh should be avoided (4).

Other sites include the upper arm (the posterior midthird of the arm between the shoulder and elbow joint) and the buttock (upper outer quadrant). The upper arm is preferred for social and practical reasons, especially when insulin is administered by a medical professional, family member or self- injected in public. The buttocks are used in toddlers and younger children.

Since absorption varies from site to site, an injection at a certain hour should be given in the same anatomical site to enable patients to predict the effect of a given dose (6). However, injection sites must be rotated systematically each day to maintain skin health, to avoid lipo-hypertrophy and to optimize insulin absorption. Injections should be spaced 1-2 cm apart in order to minimize tissue trauma.

\section{Using a syringe: One insulin, one vial}
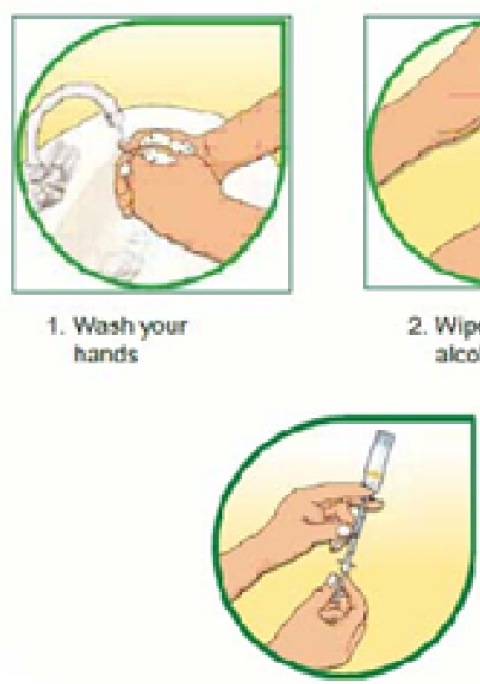

5. Tum the insuln bottle and sringe upside down

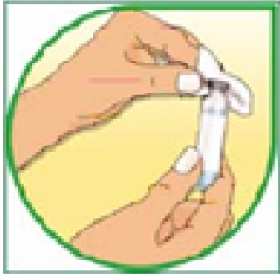

2. Wipe with aledhol sugh

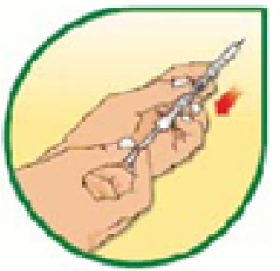

3. Draw air equal to the amount of ineulin to be whoran

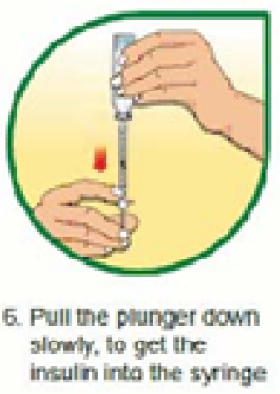

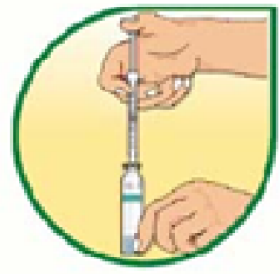

4. Push the needle through the stopper or the vio

Figure 1. Injection preparation. 


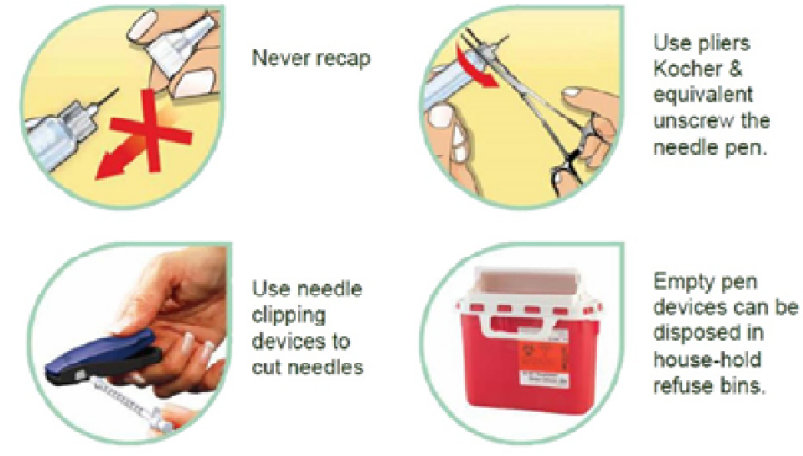

Figure 2. Safe disposal of injection devices.

\section{Selection of needle length}
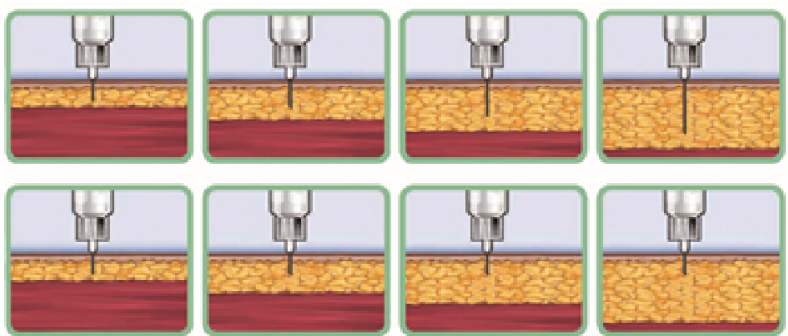

Figure 3. Needle length in specific patient population.

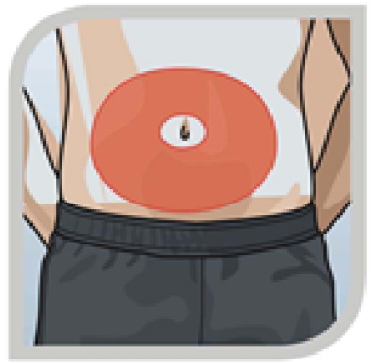

Abdomen

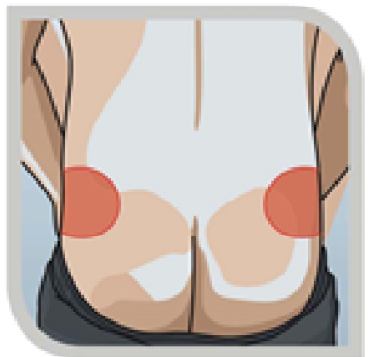

Buttocks

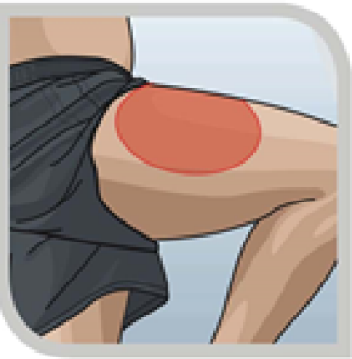

Thigh

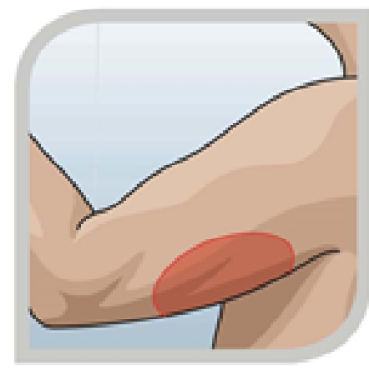

Arms
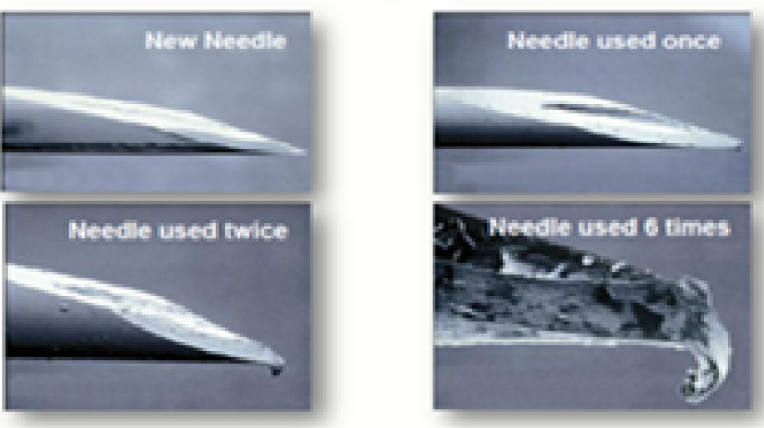

Figure 6. Needle reuse causes damage to the tip of the needle.

\section{Injection site preparation}

The chosen injection site may be cleaned with cotton balls dipped in water or with alcohol swabs, prior to injection. If the site is 'socially clean', i.e., one is willing to touch the skin, there is no need for extra cleansing (4). Soap-based detergents should be avoided as far as preinjection cleansing is concerned.

\section{Injection preparation}

The insulin device should be checked for expiry date, possible damage, clumping, frosting or precipitation, prior to use (7). It should be brought to room temperature by keeping it outside the refrigerator for 30 minutes (4).

If using a syringe and vial, one must wipe the top of the vial with an alcohol swab, draw air into the syringe and push it in the vial, and then draw the insulin dose required, while holding the bottle upside down. The syringe should be checked for air bubbles prior to injection (8).

If insulin preparations have to be mixed, regular insulin should be filled first, followed by NPH insulin, so that no protamine contaminates the regular insulin vial (7). Glargine cannot be mixed with any other insulin preparations, because of its' low $\mathrm{pH}$. 


\section{Table 4. Good injection practices (GIP) to minimize pain associated with insulin}

\section{Pre-injection}

O Appropriate messaging: convey the benefits of insulin in a positive manner

O Appropriate site selection and preparation

O Appropriate device selection

- Thin gauge needles

- Short needles

- Pens requiring less pressure

- New needle for each injection

O Appropriate dose selection:

- Consider splitting doses in persons with high insulin requirement

\section{During injection}

- Allow insulin to reach room temperature before injecting

- Allow alcohol to dry before injecting insulin

- Do not raise a tight painful or blanched skin fold

- Inject slowly

- Avoid injecting at hair roots

- Avoid intramuscular and intradermal injections

- Avoid injecting over bruised or traumatized sites

- Do not move the needle while injecting

- Follow systematic rotation policy

\section{Post injection}

- Release skin fold(if raised earlier) slowly

- Do not massage the injection site

- Feel happy: insulin is a life saver

While using insulin pens, whether reusable or disposable, the first step is to prime the pen with two units of insulin. The appropriate dose should be dialed prior to injecting and this can be checked on the display window of the device (4).

\section{Minimizing pain}

Some patients complain of pain with insulin injections. Unless the needle irritates a nerve ending, true pain is rare and it may be a manifestation of needle phobia, insulin phobia, or negative conditioning due to prior experiences. Good injection practices, which can minimize injectionassociated pain, are listed in Table 4.

\section{Trouble shooting}

As with any medical intervention, insulin administration may be associated with minor complications. Lipohypertrophy is a localized increase in size of adipocytes, which occurs at sites of repeated insulin injections and reuse of needles may precipitate this.

Though patients tend to reuse lipohypertrophic sites because of lowered sensitivity to pain, they should be avoided as absorption of insulin can be erratic. Systematic rotation policy, avoiding reuse of needles and avoiding injections in hypertrophied sites help to prevent and manage lipohypertrophy $(4,7)$.

Other local site reactions include bleeding and bruising, which are usually self-limiting, and amyloidosis (9).

Needle stick injuries are another complication of injectable therapy, which can be minimized by following basic needle hygiene and injection disposal policies. Injection through clothing must be strongly discouraged, as also the injections over the forearm or calf muscles.

\section{Disposal}

Appropriate disposal of insulin injections and related accessories is an important, yet often neglected aspect of insulin technique. Inappropriate disposal may lead to needle stick injuries and spread of blood- borne infections, in people with diabetes their family members, health care professionals, and sanitary workers who collect and recycle trash or garbage $(4,7)$. Dedicated sharps collection and disposal devices are ideal. A practical and safe method of disposing needles and lancets is to collect them in a large glass container (for example, a used intravenous fluid bottle) and seal the container tightly before disposing it off in non-recyclable trash bins.

\section{Conclusion}

Insulin technique is an integral part of diabetes therapy and it is as important as the insulin prescription itself. There is a growing realization that incorrect insulin technique is often the reason for sub optimal response to insulin therapy. Inappropriate technique may cause harm to the patient, in form of unexpected hypoglycemia, uncontrolled hyperglycemia, local site reactions, pain and needle stick injuries. It may also harm other people, if good sharps disposal practices are not followed. Ongoing 
training, audit and improvement in insulin injection practices are required in order to ensure optimal insulin delivery.

\section{References}

1. Keininger D, Coteur G. Assessment of self-injection experience in patients with rheumatoid arthritis: psychometric validation of the Self-Injection Assessment Questionnaire (SIAQ). Health Qual Life Outcomes 2011; 9: 2 .

2. Bahrmann A, Abel A, Zeyfang A, Petrak F, Kubiak T, Hummel J, et al. Psychological insulin resistance in geriatric patients with diabetes mellitus. Patient Educ Couns 2014; 94: 417-22.

3. Abu Hassan H, Tohid H, Mohd Amin R, Long Bidin MB, Muthupalaniappen L, Omar K. Factors influencing insulin acceptance among type 2 diabetes mellitus patients in a primary care clinic: a qualitative exploration. BMC Fam Pract 2013; 14: 164.
4. Kalra S, Balhara YP, Baruah MP, Chadha M, Chandalia HB, Chowdhury S, et al. Forum for injection techniques, India: the first Indian recommendations for best practice in insulin injection technique. Indian JEndocrinol Metab 2012; 16: 876-85.

5. Frid A, Hirsch L, Gaspar R, Hicks D, Kreugel G, Liersch J, et al. New injection recommendations for patients with diabetes. Diabetes Metab 2010; 36(2): 3-18.

6. King L. Subcutaneous insulin injection technique. Nurs Stand 2003; 17: 45-52.

7. American Diabetes Association. Insulin Administration. Diabetes Care 2004; 27(1);106-9.

8. Kansra UC, Sircar S. Insulin therapy: Practical points. $J$ Indian Acad Clin Med 2000; 1:285-93.

9. Shikama Y, Kitazawa J, Yagihashi N, Uehara O, Murata Y, Yajima N, et al. Localized amyloidosis at the site of repeated insulin injection in a diabetic patient. Intern Med 2010; 49: 397-401. 